\title{
Schedule factors in the emission of distress calls'
}

HOWARD S. HOFFMAN AND JAMES W. STRATTON THE PENNSYLVANIA STATE UNIVERSITY
When ducklings have been imprinted to a moving stimulus, subsequent withdrawal of the stimulus usually leads to the emission of distress calls. Four Peking ducklings were imprinted and tested for the emission of distress calls under each of four different schedules of stimulus presentation and withdrawal. Although the total time for presentation and withdrawal was equal for the several schedules, fewer distress calls were emitted during short cycles of presentation and withdrawal than during long cycles.

With the immature duckling, as with the infant of many species, the departure of the mother typically leads to the emission of a stream of loud distresslike vocalizations which terminate with her return (Hafez, 1962; Sluckin, 1965). The present investigation was concerned with the way in which the schedule of the mother's withdrawal and return influences these calls. If, for example, the mother can be present for only 10 out of $20 \mathrm{~min}$, does it make any difference if she withdraws and returns in five cycles of $2 \mathrm{~min}$ present and $2 \mathrm{~min}$ withdrawn or in 10 cycles of $1 \mathrm{~min}$ present and $1 \mathrm{~min}$ withdrawn? If the duckling's tendency to emit distress calls is determined by total contact time, then both schedules should yield equivalent amounts of distress calling. If, on the other hand, schedule factors play a role, then the two sequences should yield different amounts of calling. The present work sought to evaluate this question by imprinting ducklings to a moving stimulus and then tabulating the distress calls engendered when the imprinted stimulus was subsequently presented and withdrawn according to each of several controlled sequences.

Subjects

The Ss were four Peking ducklings (Anas platyrhynchos) that were hatched in isolation and maintained in individual brooder boxes with a $40 \mathrm{~W}$ light bulb for heat. The Ss had continuous access to food and water.

Apparatus

Two units were used for imprinting and subsequent test procedures. These were housed in individual sound-treated and temperature controlled rooms. Each unit consisted of a plywood box (183 x $76 \times 76 \mathrm{~cm})$ divided lengthwise by a fine-mesh brass screen into two approximately equal compartments, one for the duckling and the other for the imprinting stimulus. The imprinting stimulus consisted of a white plastic milk bottle mounted over the superstructure of a model train engine which ran the length of its compartment on HO-gauge track. The lighting in the S's compart- ment was such that when the stimulus compartment was darkened, reflections from the screen prevented $S$ from viewing the stimulus or the stimulus compartment. When, however, the stimulus compartment was illuminated, the stimulus compartment and its contents became visible. Stimulus presentation consisted of illuminating the stimulus compartment and moving the stimulus at approximately $30 \mathrm{~cm} / \mathrm{sec}$ back and forth along its track. Stimulus withdrawal, on the other hand, consisted of darkening the stimulus compartment and terminating stimulus movement.

Distress calls were detected with specially constructed voice keys which, through selective filtering, were sensitive only to those sounds in the frequency range within which distress vocalizations typically fall (approximately $3000-4000 \mathrm{cps}$ ). ${ }^{2}$ A bank of relays, stepping switches, timers, and recorders was employed to program stimulus presentations and to record distress calls.

\section{Procedure}

All ducklings were exposed individually to four imprinting sessions during the first $48 \mathrm{~h}$ after hatch. Each session lasted $45 \mathrm{~min}$. The duckling was placed in the runway with the stimulus compartment illuminated and the stimulus moving back and forth along its track. The duckling remained in this condition for the entire session. Tests for the effects of the several schedules occurred on the fifth and sixth days after hatching.

Each of these tests employed one of the following schedules of stimulus presentation and withdrawal: 108 cycles of $3 \mathrm{sec}$ present, $3 \mathrm{sec}$ withdrawn; 36 cycles of 9 sec present, 9 sec withdrawn; 12 cycles of 27 sec present, 27 sec withdrawn; 4 cycles of 81 sec present, 81 sec withdrawn. With this arrangement all schedules incorporated a total of 324 $\mathrm{sec}$ of stimulus presentation and $324 \mathrm{sec}$ of stimulus withdrawal.

Each duckling was exposed to all four schedules on each of the two test days. On a given day each schedule was presented in a separate test session with the interval between test sessions approximately $2 \mathrm{~h}$. On each day the sequence of schedules was counterbalanced across ducklings by arranging the sequence according to a Latin Square. A given test session was preceded by a $4 \mathrm{~min}$ adaptation period during which the imprinted stimulus was visible and moving. The session began with the initial removal of the stimulus and terminated after the final stimulus presentation. 


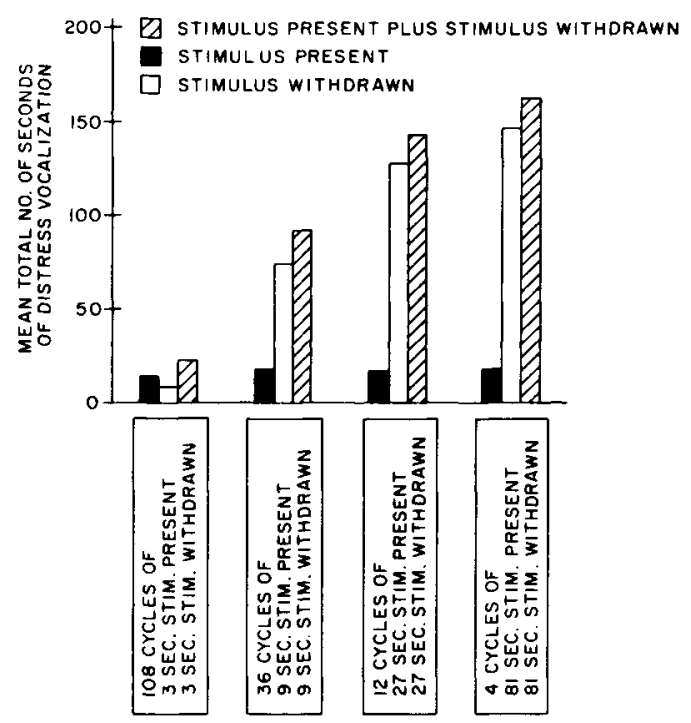

Fig. 1. Mean total number of seconds of distress calls per test session during each of four schedules of stimulus presentation and withdrawal. With each of these schedules the imprinted stimulus was present for 324 out of a total of $648 \mathrm{sec}$

\section{Results and Discussion}

Figure 1 shows the mean (across Ss) of the total number of seconds of distress vocalization per test session when the imprinted stimulus was present and when it was withdrawn. It also shows the totals across the two stimulus conditions. A repeated measures analysis of variance (Winer, 1962) of these totals yielded $F=12.73$, df $=3 / 9, p<.01$; hence it can be concluded that the large observed differences among schedules are statistically reliable. As seen in Fig. 1, during stimulus prsentation very few distress calls were recorded, and in general the emission of these calls did not vary with the schedules. During stimulus withdrawal, on the other hand, ducklings vocalized more or less continuously during extended withdrawal of the stimulus, but few calls were emitted when the periods of stimulus withdrawal had a duration of only 3 sec.

Since all four schedules incorporated $324 \mathrm{sec}$ of stimulus presentation and $324 \mathrm{sec}$ of stimulus withdrawal, the differences in distress vocalization must be attributed to schedule factors rather than to overall exposure time. The low incidence of distress vocal- ization during the schedule involving 3 sec cycles of stimulus presentations makes it clear that the act of stimulus withdrawal is not itself a sufficient factor in eliciting distress calls. If it were, one would expect most calls during 3 sec exposure cycles since that condition maximized the number of stimulus withdrawals.

An interpretation of the present findings can be derived from results of earlier studies in this laboratory (Hoffman et al, 1966a; Hoffman et al, 1966b). Those studies indicated that although distress calls usually stop abruptly with stimulus presentation, withdrawal of the stimulus is seldom accompanied by the immediate onset of distress vocalization. Rather, the duckling remains silent during stimulus departure, and only after several seconds have elapsed do distress calls begin to appear. Apparently, when the imprinted stimulus departs and returns in $3 \mathrm{sec}$ cycles, the departure time is too short to permit the full development of the conditions responsible for distress vocalization; whereas the periods of stimulus presentation are long enough to counteract such conditions as do develop. As a result, the duckling vocalizes very little, if at all, even though across an entire session the imprinted stimulus is only present $50 \%$ of the time.

\section{References}

HAFEZ, E. S. E. The behavior of domestic animals. London: Bailer, Tindall, and Cox, 1962.

HOFFMAN, H. S. The control of distress vocalization by an imprinted stimulus. Behaviour. In press.

HOFFMAN, H. S., SCHIFF, D., ADAMS, J., \& SEARLE, J. L. Enhanced distress vocalization through selective reinforcement. Science, 1966a, $151,352-354$.

HOFFMAN, H. S., SEARLE, J. L., TOFFEY, S., \& KOZMA, F., JR Behavioral control by an imprinted stimulus. J. exp. A nal Behav., $1966 \mathrm{~b}, 9,177-189$.

SLUCKIN, W. Imprinting and early learning. Chicago: Aldine Publishing Company, 1965 .

WINER, B. J. Statistical principles in experimental design. New York: McGraw-Hill, 1962.

\section{Notes}

1. Supported by NIMH Grant MH 12824-01. We thank John Searle for his assistance and helpful suggestions.

2. In order to insure that the voice keys would selectively respond to distress calls and not to other sounds, several pilot Ss were placed in the apparatus and repeatedly captured and released. During capture, Ss typically emitted a constant stream of distress calls, and the voice key consistently responded to them. Movements of the imprinted stimulus, however, and other sounds of the Ss (for example, the low-intensity contentment cheep) never activated the voice key. 